\title{
26. Effect of anxiety disorder and depression in postoperative pain
}

\section{Sofia Poulida ${ }^{1, *}$, Lucia Makra $^{1}$}

${ }^{1}$ Department of Anaesthesiology, General Hospital of Mitilini "Vostanion", Mitilini, Lesvos, Greece. * sophia_poulida@hotmail.com

Introduction: The aim of this study is to briefly present and to evaluate the elements that show whether the psychological background of the patients, particularly anxiety disorder and depression, are involved in the mechanism of postoperative pain, to examine the mechanisms involved in the intensity of pain and to report the methods used to evaluate and treat pain after a surgical procedure in this vulnerable group of patients.

Methods: We performed a literature review of relevant articles, mainly published during the last decade, in MEDLINE database and 15 articles were used.

Results: Clinical studies lead us to the conclusion that the intensity of postoperative pain is directly related to the levels of anxiety and to the presence of depression. Undertreatment and delay in managing acute postoperative pain can lead to the development of chronic pain syndromes with consequent negative effects in life and in the level of functionality of patients. A number of neurobiological processes could further explain the effect of psychological factors on pain, especially after surgical procedures.

Conclusion: The psychological aspect of acute postoperative pain can be evaluated during the preoperative period, in order to relieve the intense negative psychological experience of pain after surgery, by using pharmacological therapy as well as psychotherapy. It is of great importance to perform psychological monitoring of patients after surgery, since it has been observed that the psychological phenotype of patients is altered during the first postoperative days. Catastrophology is involved in elevated intensity of postoperative pain, therefore the appropriate use of relevant clinical interventions would be beneficial. Psychosocial and psychophysiological evaluation is of great importance in order to detect patients at high risk and to offer individualized management and targeted preventive preoperative planning. The heterogeneity of patient population going to surgery increases the need for further studies which would evaluate appropriate therapeutic regimens, individualized for each special group of patients. 